\title{
The Relationship between Bereaved Family Caregivers and the Hospital Team in Long Term Care: Cases of Home Condolence Visits
}

\author{
Efraim Jaul ${ }^{12^{*}}$, Madlena Spivak ${ }^{1}$, Yonit Zabari $^{2}$ and Menachem Monnickendam ${ }^{3}$ \\ ${ }^{1}$ Skilled Geriatric Nursing Department, Herzog Hospital, Jerusalem, Israel \\ 2Department Herzog Hospital, Jerusalem, Israel. affiliated with the Hebrew University Medical School, Jerusalem, Israel \\ ${ }^{3}$ The Louis and Gabi Weisfeld School of Social Work. Bar Ilan University, Israel
}

"Corresponding author: Efraim Jaul, MD, MPH, Director, Department of Skilled Nursing, Herzog Hospital, PO Box 3900 , Jerusalem 91035, Israel, Tel: +972-52-8300075; Fax: +972-2-653-6075; E-mail: jaul@zahav.net.il

Rec date: Jan 28, 2015; Acc date: Apr 27, 2015; Pub date: Apr 30, 2015

Copyright: ( 2015 Jaul E, et al. This is an open-access article distributed under the terms of the Creative Commons Attribution License, which permits unrestricted use, distribution, and reproduction in any medium, provided the original author and source are credited.

\begin{abstract}
Condolence visits are an integral part of the therapeutic medical process and contribute to the comprehensive treatment of the "whole" patient. This article describes the spontaneous evolvement of condolence visits by team members of an geriatric skilled nursing department and discusses the ensuing issues and questions as a result.

Method and participants: The primary medical team consists of physicians, nurses, and a social worker of the Herzog Geriatric Skilled Nursing Department in Jerusalem, Israel, conducted home condolence visits of deceased patients' families. Inclusion criteria for the study included: 1) at least two week admission and either 2) close knit relationship, more than 10 discussions between staff and family or 3) unique cases in which death was unexpected. 4) The families where the spouse or offspring as the primary caregivers.
\end{abstract}

In this retrospective study the authors initiated a series of discussions with the medical team members who cared for these patients and participated in condolence visits. The discussions focused on understanding the underlying reasons for the occurrence of the spontaneous condolence visits and ensuing issues and questions.

Results and discussion: Fifteen cases are included in this study. The primary caregiver was the wife (4), offspring (11), mainly female. Most families included were due to the close knit relationship between family and staff.

The recurring themes of the discussion were: Do these visits invade the privacy of the families during the initial mourning period? Are the home visits really a part of the professional treatment? Are these visits confusing the professional and personal boundaries? What purposes do these visits serve? Do these visits benefit the families and lor the staff?

Conclusions: Given that the condolence visits are beneficial to team members and probably also to the bereaved family we discuss how should such visits be conducted?

Keywords: Home condolence visits; Healthcare process; Medical team; Comprehensive and multi-Disciplinary approach; Bereavement

\section{Introduction}

Providing optimal care for the elderly patient requires comprehensive care, incorporating a multi-disciplinary team and coordination with professionals of several disciplines [1]. The evaluation is accomplished with an emphasis upon the whole person, patient-centered and not organ-centered, and with a focus on the family [2]. The caring family is a unique contribution to the medical treatment process, and its importance is not to be underestimated. The elderly population relies upon the families both in the physical (instrumental tasks and self-care) domain, and also in the emotional and spiritual realm [3]. Medical teams who disregard the family influence and participation ignore one of the main caring components of medical and related health treatment.
The initial idea to visit the grieving family in order to provide closure arose when the staff noticed that the death of a patient, often suddenly or in the middle of the night, caused an abrupt cessation of encounters with the family. The medical staff feels the need to continue to meet with the family in order to help provide closure. The death, in itself, does not cause the cutoff of the relationship; rather the abrupt cessation of visits by daily family members caused feelings of abandonment and unresolved therapeutic relationship. The following example can clarify the issue. A patient with advanced dementia and pressure ulcers was hospitalized for an extended period of time in the geriatric skills unit. He received daily visits by his wife, which provided multiple opportunities for interaction with the medical staff and the development of a significant relationship. The couple had no children. This is a potential factor that increased the caregiver's dependency on the medical staff and strengthened the relationship.

Numerous questions concerning minute details were posed by the wife who visited daily, fearing that the patient will deteriorate or die in her absence, and queries by the daughters who were intensively 
involved in the care of the patient. These family members were closely involved with the staff, exhibiting anxiousness, dependency or imminent abandonment and compensating by frequently demanding attention by the staff [4]. The family-staff close relationship developed during the ongoing visits.

There are numerous encounters with the patient's family, especially those patients in long-term care (LTC). The final and perhaps most meaningful encounter with the family in the medical process occur after the patient's death. The relevance of dealing with the patient's death in LTC focuses on the team visit with the family and having a meaningful interaction in order to provide closure, as opposed to the team sending a condolence letter, calling the family on the phone to express the condolence sentiments, attending the patient's funeral, or after-care condolence follow up, individually or group sessions [5-8]. The medical staff believes that the visit to the grieving family constitutes an integral part of the caring process, and thus gives closure to the care of the patient.

A number of questions arise as a result of condolence visits. In some cultures the condolence visit could be interpreted as infringing upon the privacy of the family. Do these visits invade the privacy of the families during the initial mourning periods? Are the home visits really a part of the professional treatment? Are these visits confusing the professional and personal boundaries of the staff? What purposes do these visits serve? Do these visits benefit the family andlor the staff? Finally, how should such visits be conducted?

This article will describe the medical and health care process of meeting the patient and family, continuing the relationship and treatment, ending with the closing encounter during the team home visit after death.

\section{Methods and Participants}

The Herzog Hospital Geriatric Skilled Nursing Department located in Jerusalem, Israel, is composed of 36 beds and provides care on an intermediate/long term basis for elderly patients. Included in the medical conditions is advanced dementia with pressure ulcers, swallowing problems requiring feeding tubes, tracheostomies, end stage renal failure requiring hemodialysis, continuous oxygen therapy, non-invasive ventilation and care of tracheostomy, and palliative care of oncologic and non-oncologic diseases. The majority of the patients suffer from limited function and impaired cognition. The median survival of these patients is four months - a striking contrast in comparison with acute departments in general hospitals where the duration of hospitalization is typically brief (days), episodic and in acute stages. The medical team is composed of 3 physicians, 1 nurse practitioner, 12 nurses, 12 nursing assistance, a social worker and the participation of a multidisciplinary team from the rehabilitation department. Upon admission, routine procedures include initial meetings with individual medical team members. Due to the patient's limited ability to communicate, the family caregiver during the first week meets with the medical staff, both separately and within a multidisciplinary team approach, to review and coordinate expectations as well as evolving the treatment plan. End of life issues are discussed with the family and personal desires are explored.

After death, a staff meeting is held in order to decide whether a condolence visit shall be conduct and which team members wish to participate.
Prior to the visit, the social worker coordinates the arrangement with the family. After the condolence visit, the participating staff members routinely conduct a meeting for the purpose of discussing the visit, the medical process, the family interaction, and recommendations for future treatment in similar cases. Inclusion criteria for the study included: 1) at least two week admission and either 2) close knit relationship, more than 10 discussions between staff and family over a two year period or 3) unique cases in which death was unexpected. 4) The families where the spouse or offspring as the primary caregivers.

\section{Result}

Fifteen cases are included in this study. The development of a significant and close-knit relationship between the family and the medical staff (13 cases); and unique cases in which death was unexpected ( 2 cases).

Gender distribution for the deceased consisted of 10 males and 5 females. The mean age of the deceased was 82.4 years. The principal person for the family caregiver connection consisted of 4 wives for the deceased males and 11 children (particularly daughters) for all of the deceased. The majority of the patients lived in the Jerusalem municipality and three resided at the outskirts of the city. The principal caregiver in the family was female, either wife or daughter. All the deceased died from non-oncologic diseases including: complication of advanced dementia, pressure ulcers, recurrent aspiration pneumonia and cardio-vascular problems [9].

\section{Discussion}

In this retrospective study the authors initiated a series of discussions with the medical team members who cared for these patients and participated in condolence visits. These were unstructured open discussions during which the researchers attempted to understand the underlying reasons for the occurrence of the spontaneous condolence visits and ensuing issues and questions. The main recurring themes are presented and discussed.

Do these visits invade the privacy of the families during the initial mourning periods?

In some cultures condolence visits could be interpreted as infringing upon the privacy of the family. In Judaism, as well as in other religions and cultures, it is customary to pay a condolence visit during the first week following the death. The bereaved - the closest family members - are all together, sitting on a low chair which indicates they are in mourning. The visitors which include relatives, friends and neighbors, come and give support and strength. The Shiva condolence consists of one week (Shiva in Hebrew means Seven) and is meant to process the condolence intensity, and is certainly an accepted cultural and religious tradition [10].

\section{Are the home visits really a part of the professional treatment?}

The underlying supposition is that the condolence visits are part of the continuity of the medical staff treatment process. Ferris, et al. [11] reported a survey at two academic medical centers that the house staff task of care after a death including follow up with the family [11]. Chau, et al. [6] presented a study at the annual meeting of the American Society of Clinical Oncology pertaining to condolence practices among Canadian cancer physicians. The study groups were divided into medical oncologists (MO), radiation oncologists (RO) and palliative care (PC) professionals. The frequency of the physician 
consistently sending cards were (in percent): 18.2 (MO): 7.1 (RO): 29.5 (PC) respectively. Telephoning to the family achieved a higher level of participation by all physicians: 21.1 (MO), 13.5 (RO), 34.8 (PC). Attending the funeral had the lowest level of attendance: 6.4 (MO), 3.3 (RO), 13.4 (PC). Referring family to condolence support programs had mixed priorities: 6.5 (MO), $3.9(\mathrm{RO}), 19.1(\mathrm{PC})$ as did post-terminal death family meetings: $6.4(\mathrm{MO}), 3.3(\mathrm{RO}), 13.4(\mathrm{PC})$ respectively. It is significant that these condolence practices are considered and undertaken more frequently with palliative care physicians. Participation notably is beginning to be initiated with medical oncologists and radiation oncologists. Thus it is important to recognize that the treatment process does not end with the death of the deceased, and continues through the condolence visit of the medical staff during the first week of the formal mourning period [5].

Are these visits confusing the professional and personal boundaries?

In coping with the loved one's illness, the patient's family depends in many ways upon the support and intervention from the treating physician and medical staff [12]. Treatment questions are addressed to the nursing staff and difficulties related to emotional concern are directed to the social worker. The medical conditions relating to specific acute problems (fever, vomiting, infection, convulsion etc.) are discussed with the physician. All these efforts reinforce the family affinity and attachment with the staff.

Issues surrounding death (DNR decisions, intubation and tube feeding), and personal preference frequently arise in discussion between the staff and families of the patients [13]. After the patient's death, it is often overlooked that the family still looks to the medical team for comfort, insight and guidance. A condolence visit consisting of the combined team approach by the physician, nurse and social worker to the family's home has significantly greater impact than a visit by only one staff member. The medical staff appears at the family home condolence visit in uniform, directly from work and during working hours. Such a visit is not a social call, nor is it private in nature but serves to represent the department or even the hospital in its entirety.

\section{What purposes do these visits serve?}

The main purpose of visiting the bereaved family is to transfer an awareness of empathy, recognition and contribution. Additionally, the staff attempts to relieve the family of emotions of guilt or anger that may exist by reinforcing the validity of the medical decisions that were made by the family as well as respecting the devotion and dedication of the spouse, children and family [7].

The visits express caring and concern, and help diffuse the family's closeness towards the medical team. It is important to recognize that the primary goal of the condolence visit to the home of the bereaved families is to meet the needs of the family, even though there may be personal reactions and requirements by part of the staff concerning the patient and the family $[14,15]$.

A face-to-face visit with the family facilitates: 1. Non-verbal expressions of sympathy which are impossible to communicate by any other means. 2. Allowing the family to speak amiably, with additional detail, and in a non-threatening environment. 3. Providing guidance for condolence support programs.

Do these visits benefit the family and/or the staff?
The medical staff believes that these visits constitute an integral part of the caring process for the patient's family. A visit to the grieving family thus gives closure to the care and concludes the responsibility of the staff to the patient and the family [16]. The sense of loss and failure for physicians and staff does occur as a result of the patient's death and the family lack of contact. This is experienced less keenly by geriatric physicians and specialized staffs recognizing death as a natural process and thus these professionals continue to be involved during the condolence period [17].

The home visit sets an example for a comprehensive approach by the entire medical team, emphasizing humanistic and holistic treatment and does not focus solely on technical clinical duties with the patient and family [18]. In an era of technological life supports, the entire dying process is prolonged, and places a greater emotional burden and requirements upon the family. Therefore, humanistic and comprehensive treatment who met the needs of the family and focus on the family is optimal.

In the past, the departmental policy following the demise of a patient consisted of the social worker and/or the head physician of the department communicating sympathy by telephone to the family of the deceased. Acknowledgement emerged for the necessity to change this procedure as a visit in person is substantially greater in impact than a mere phone call.

In a telephone call conducted among two third of the bereaved families a month after the patient's demise, the families expressed profound appreciation for the deceased treatment and for the condolence visit by the medical team.

\section{What criteria should be applied to decide which families to visit?}

Primary and secondary criteria for condolence visits have now been established. Although visiting the families of all deceased patients would be ideal, it is unrealistic (time, absence of staff and distance) to expect staff to make condolence visits for all deceased patients [19].

1. The primary criteria chosen established the acceptance and awareness of a significant and close-knit relationship developed between the family and the staff, and thus it is deemed appropriate for continuity and closure to make the home visit. The nature of a deeper relationship with the family includes open expression of feelings during the hospitalization of the patient, and frequent, lengthy conversations conducted with the staff. Furthermore, the probability of a close relationship between the medical team and the family is abundantly greater when the dying process has extended over a long period.

2. The secondary criteria utilized were to clarify the cause of unexpected death, respond to complaints, anger and/or dissatisfaction expressed by the family.

For example, the patient may have died suddenly and unexpectedly, the family may not have been prepared and perhaps they may suspect that there were influencing factors, including neglect or lack of attention pertaining to the sudden death. A home visit during the first week after the patient's death gives the family a chance to inquire and clarify the cause of the death and to vent suspicions or anger on a faceto-face basis with the staff.

\section{How should such visits be conducted?}

- Listen to the family relates the loved one's life story, and initially allow the family to lead the conversation. The staff preferably, 
whenever possible, shares positive impressions about the patient with the family.

- Verbalize empathy with the family's grief and depth of loss. Empathy with the family encourages the family to vent emotions and convictions as a healing process.

- Convey to the family that caring for the loved one is a privilege and not only a responsibility.

- Undertake an effort to determine whether there are guilt feelings on the part of the family. The staff may respond by saying "You did everything you could for your father" or "Your mother would have died much sooner without your dedication." Other comforting statements are "He is no longer suffering" and "He didn't suffer during the process of dying."

- Staff compliments the family for dedication during the deceased's lifetime, providing a desirable example for grand-children and other relatives to honor and act properly with a parent. An opportunity is also provided for the family to express and convey appreciation to the medical team.

- The staff maintains caution and sensitivity with regards to the family's emotions reactions. Avoid using phrases such as: "You'll get over it" or "Everything will be fine". Do not "lecture" on coping with the situation nor discuss the staff's own personal losses.

\section{Conclusion}

The visit of the medical team recognizes and reinforces awareness that the family role is acknowledged in the dying process and that the need for assistance continues throughout the grieving process. The medical team utilizes this occasion as a means for assisting the family to attain closure, providing both medical information and direction for additional support and condolence counseling.

A condolence visit to the grieving family by the physician and the medical team is considered to be an integral part of continuity for the patient and family. Visiting the grieving family includes a positive ripple effect on the relationship between the medical team and other families in the hospital setting. The goal is to complete the dying process affecting the family in a way that enables individuals to return to routine life.

These visits are particularly appropriate, desirable and acceptable by the families with patients in long-term care wards as the medical team relationship with the family is intensive both in terms of duration and depth.

The process of the consolation home visit enables a new perspective of comprehensive and distinguishes professional work going beyond the technical clinical treatment of the patient and includes the family and community as essential elements.

A new hospital-wide policy statement regarding condolence visits is advisable, and importance placed in developing written departmental protocols for this activity and other condolence procedures.

Condolence visits are an integral and important part of the therapeutic medical process and viewed as necessary for comprehensive treatment of the "whole" patient. A pivotal role with family members and relatives to terminate the process is vital and provides valuable continuity and closure for both the staff care and the families as well.

\section{Acknowledgement}

I would thank Olga Shirov RN, and Ronit Behar- Goldberger Nurse Practitioner Herzog Hospital Jerusalem, Israel, for participation in the condolence visits.

\section{References}

1. Musil CM, Warner CB, Saeid H (2003) Issues in Care-givres' Stress and Providers'. Support. Research on Aging 25: 505-526

2. Hannum Rose J, Bowman KF, O'Toole EE, Abbott K, Love TE, et al. (2007) Caregiver objective burden and assessments of patient-centered, family-focused care for frail elderly veterans. Gerontologist 47: 21-33.

3. Glasser M, Prohaska T, Gravdal J (2001) Elderly Patients and their Accompanying Caregivers on Medical Visits. Research on Aging 23: 326-348.

4. Kramer BJ, Kipnis S (1995) Eldercare and work-role conflict: toward an understanding of gender differences in caregiver burden. Gerontologist 35: 340-348.

5. Wolfson R, Menkin E (2003) Writing a condolence letter. J Palliat Med 6: 77-78.

6. Tolle SW, Bascom PB, Benson JA, Hickam DH (1986) Communication between physicians and surviving spouses following patient deaths Journal of General Internal Medicine 1: 309-314

7. Chau N, Zimmerman C, Tabak MN (2008) Brevement practices of Candian cancer physician. J of Clinical Oncology 26: 9550.

8. Coolican MB, Pearce T (1995) After care bereavement program. Crit Care Nurs Clin North Am 7: 519-527.

9. Songwathana P (2001) Women and AIDS caregiving: women's work? Health Care Women Int 22: 263-279.

10. Maimonides, Mishneh-Torah, Sefer Shoftim, Hilchot Aveilim 13: 1.9

11. Ferris TG, Hallward JA, Ronan L, Billings JA (1998) When the patient dies: a survey of medical housestaff about care after death. J Palliat Med 1: 231-239.

12. Milberg A, Strang P (2011) Protection against perceptions of powerlessness and helplessness during palliative care: the family members' perspective. Palliat Support Care 9: 251-262.

13. Jaul E, Zabari Y, Brodsky J (2014) Spiritual background and its association with the medical decision of, DNR at terminal life stages. Arch Gerontol Geriatr 58: 25-29.

14. Kearney MK, Weininger RB, Vachon ML, Harrison RL, Mount BM (2009) Self-care of physicians caring for patients at the end of life: "Being connected... a key to my survival". JAMA 301: 1155-1164, E1.

15. Aiken LH, Clarke SP, Sloane DM, Sochalski J, Silber JH (2002) Hospital nurse staffing and patient mortality, nurse burnout, and job dissatisfaction. JAMA 288: 1987-1993.

16. Penson RT, Green KM, Chabner BA, Lynch TJ Jr (2002) When does the responsibility of our care end: bereavement. Oncologist 7: 251-258.

17. Moores TS, Castle KL, Shaw KL, Stockton MR, Bennett MI (2007) 'Memorable patient deaths': reactions of hospital doctors and their need for support. Med Educ 41: 942-946.

18. Tolle SW, Cooney TG, Hickam DH (1989) A program to teach residents humanistic skills for notifying survivors of a patient's death. Acad Med 64: 505-506.

19. Jaul E (2010) The physician and medical staff home condolence visit. J Palliat Med 13: 640 . 